\title{
Erratum to: Measurement of jet production cross sections in deep-inelastic ep scattering at HERA
}

\section{H1 Collaboration}

V. Andreev ${ }^{19}$, A. Baghdasaryan ${ }^{31}$, K. Begzsuren ${ }^{28}$, A. Belousov ${ }^{19}$, A. Bolz ${ }^{12}$, V. Boudry ${ }^{22}$, G. Brandt ${ }^{41}$, V. Brisson ${ }^{21}$, D. Britzger ${ }^{10}$, A. Buniatyan ${ }^{2}$, A. Bylinkin ${ }^{43}$, L. Bystritskaya ${ }^{18}$, A. J.Campbell ${ }^{10}$, K. B. Cantun Avila ${ }^{17}$, K. Cerny ${ }^{25}$, V. Chekelian ${ }^{20}$, J. G. Contreras ${ }^{17}$, J. Cvach ${ }^{24}$, J. B. Dainton ${ }^{14}$, K. Daum ${ }^{30}$, C. Diaconu ${ }^{16}$, M. Dobre ${ }^{4}$, V. Dodonov ${ }^{10, \dagger}$, G. Eckerlin ${ }^{10}$, S. Egli ${ }^{29}$, E. Elsen ${ }^{10}$, L. Favart ${ }^{3}$, A. Fedotov ${ }^{18}$, J. Feltesse ${ }^{9}$, J. Ferencei ${ }^{44}$, M. Fleischer ${ }^{10}$, A. Fomenko ${ }^{19}$, E. Gabathuler ${ }^{14},{ }^{\dagger}$, J.Gayler $^{10}$, S. Ghazaryan ${ }^{10}, \dagger$, L. Goerlich ${ }^{6}$, N. Gogitidze ${ }^{19}$, M. Gouzevitch ${ }^{35}$,

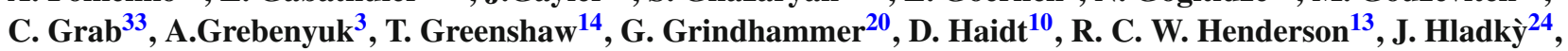
D. Hoffmann ${ }^{16}$, R. Horisberger ${ }^{29}$, T. Hreus ${ }^{3}$, F. Huber ${ }^{12}$, M. Jacquet ${ }^{21}$, X. Janssen ${ }^{3}$, H. Jung ${ }^{10,3}$, M. Kapichine ${ }^{8}$, J. Katzy ${ }^{10}$, C. Kiesling ${ }^{20}$, M. Klein ${ }^{14}$, C. Kleinwort ${ }^{10}$, R. Kogler ${ }^{11}$, P. Kostka ${ }^{14}$, J. Kretzschmar ${ }^{14}$, D. Krücker ${ }^{10}$, K. Krüger ${ }^{10}$, M. P. J. Landon ${ }^{15}$, W. Lange ${ }^{32}$, P. Laycock ${ }^{14}$, A. Lebedev ${ }^{19}$, S. Levonian ${ }^{10}$, K. Lipka ${ }^{10}$, B. List ${ }^{10}$, J. List ${ }^{10}$, B. Lobodzinski ${ }^{20}$, E. Malinovski ${ }^{19}$, H.-U. Martyn ${ }^{1}$, S. J. Maxfield ${ }^{14}$, A. Mehta ${ }^{14}$, A. B. Meyer ${ }^{10}$, H. Meyer ${ }^{30}$, J. Meyer ${ }^{10}$, S. Mikocki ${ }^{6}$, A. Morozov ${ }^{8}$, K. Müller ${ }^{34}$, Th. Naumann ${ }^{32}$, P. R. Newman ${ }^{2}$, C. Niebuhr ${ }^{10}$, G. Nowak ${ }^{6}$, J. E. Olsson ${ }^{10}$, D. Ozerov ${ }^{29}$, C. Pascaud ${ }^{21}$, G. D. Patel ${ }^{14}$, E. Perez ${ }^{37}$, A. Petrukhin ${ }^{35}$, I. Picuric ${ }^{23}$, H. Pirumov ${ }^{10}$, D. Pitzl ${ }^{10}$, R. Plačakytè ${ }^{10}$, R. Polifka ${ }^{25,39}$, V. Radescu ${ }^{45}$, N. Raicevic ${ }^{23}$, T. Ravdandorj ${ }^{28}$, P. Reimer $^{24}$, E. Rizvi $^{15}$, P. Robmann ${ }^{34}$, R. Roosen ${ }^{3}$, A. Rostovtsev ${ }^{42}$, M. Rotaru ${ }^{4}$, D. Š́lek ${ }^{25}$, D. P. C. Sankey ${ }^{5}$, M. Sauter ${ }^{12}$, E. Sauvan ${ }^{16,40}$, S. Schmitt ${ }^{10}{ }^{10}$, L. Schoeffel ${ }^{9}$, A. Schöning ${ }^{12}$, F. Sefkow ${ }^{10}$, S. Shushkevich ${ }^{36}$, Y. Soloviev ${ }^{10,19}$, P. Sopicki ${ }^{6}$, D. South ${ }^{10}$, V. Spaskov ${ }^{8}$, A. Specka ${ }^{22}$, M. Steder ${ }^{10}$, B. Stella ${ }^{26}$, U. Straumann ${ }^{34}$, T. Sykora ${ }^{3,25}$, P. D. Thompson ${ }^{2}$, D. Traynor ${ }^{15}$,

P. Truöl ${ }^{34, \dagger}$, I. Tsakov ${ }^{27}$, B. Tseepeldorjj ${ }^{28,38}$, A. Valkárová ${ }^{25}$, C. Vallée ${ }^{16}$, P. Van Mechelen ${ }^{3}$, Y. Vazdik ${ }^{19, \dagger}$,

D. Wegener ${ }^{7}$, E. Wünsch ${ }^{10}$, J. Žáček ${ }^{25}$, Z. Zhang ${ }^{21}$, R. Žlebčík ${ }^{25}$, H. Zohrabyan ${ }^{31}$, F. Zomer ${ }^{21}$

${ }^{1}$ I. Physikalisches Institut der RWTH, Aachen, Germany

${ }^{2}$ School of Physics and Astronomy, University of Birmingham, Birmingham, UK ${ }^{\mathrm{c}}$

${ }^{3}$ Inter-University Institute for High Energies ULB-VUB, Brussels and Universiteit Antwerpen, Antwerp, Belgium ${ }^{\mathrm{d}}$

${ }^{4}$ Horia Hulubei National Institute for R\&D in Physics and Nuclear Engineering (IFIN-HH), Bucharest, Romania ${ }^{j}$

${ }^{5}$ STFC, Rutherford Appleton Laboratory, Didcot, Oxfordshire, UK

${ }^{6}$ Institute of Nuclear Physics Polish Academy of Sciences, Krakow, Poland ${ }^{\mathrm{e}}$

${ }^{7}$ Institut für Physik, TU Dortmund, Dortmund, Germany ${ }^{\mathrm{b}}$

${ }^{8}$ Joint Institute for Nuclear Research, Dubna, Russia

${ }^{9}$ Irfu/SPP, CE Saclay, Gif-sur-Yvette, France

${ }^{10}$ DESY, Hamburg, Germany

${ }^{11}$ Institut für Experimentalphysik, Universität Hamburg, Hamburg, Germany ${ }^{\mathrm{b}}$

${ }^{12}$ Physikalisches Institut, Universität Heidelberg, Heidelberg, Germany ${ }^{\mathrm{b}}$

13 Department of Physics, University of Lancaster, Lancaster, UK ${ }^{\mathrm{c}}$

${ }^{14}$ Department of Physics, University of Liverpool, Liverpool, UK ${ }^{\mathrm{c}}$

${ }^{15}$ School of Physics and Astronomy, Queen Mary, University of London, London, UK

${ }^{16}$ Aix Marseille Université, CNRS/IN2P3, CPPM UMR 7346, 13288 Marseille, France

${ }^{17}$ Departamento de Fisica Aplicada, CINVESTAV, Mérida, Yucatán, Mexico ${ }^{\mathrm{h}}$

${ }^{18}$ Institute for Theoretical and Experimental Physics, Moscow, Russia ${ }^{\mathrm{i}}$

${ }^{19}$ Lebedev Physical Institute, Moscow, Russia

${ }^{20}$ Max-Planck-Institut für Physik, Munich, Germany

${ }^{21}$ LAL, Université Paris-Sud, CNRS/IN2P3, Orsay, France

${ }^{22}$ LLR, Ecole Polytechnique, CNRS/IN2P3, Palaiseau, France

${ }^{23}$ Faculty of Science, University of Montenegro, Podgorica, Montenegro ${ }^{\mathrm{k}}$

${ }^{24}$ Institute of Physics, Academy of Sciences of the Czech Republic, Praha, Czech Republic ${ }^{\mathrm{f}}$

${ }^{25}$ Faculty of Mathematics and Physics, Charles University, Praha, Czech Republic ${ }^{\mathrm{f}}$

${ }^{26}$ Dipartimento di Fisica Università di Roma Tre and INFN Roma 3, Rome, Italy

${ }^{27}$ Institute for Nuclear Research and Nuclear Energy, Sofia, Bulgaria

${ }^{28}$ Institute of Physics and Technology of the Mongolian Academy of Sciences, Ulaanbaatar, Mongolia

${ }^{29}$ Paul Scherrer Institut, Villigen, Switzerland

${ }^{30}$ Fachbereich C, Universität Wuppertal, Wuppertal, Germany

${ }^{31}$ Yerevan Physics Institute, Yerevan, Armenia 
${ }^{32}$ DESY, Zeuthen, Germany

${ }^{33}$ Institut für Teilchenphysik, ETH, Zurich, Switzerland ${ }^{\mathrm{g}}$

${ }^{34}$ Physik-Institut der Universität Zürich, Zurich, Switzerland ${ }^{g}$

35 Now at IPNL, Université Claude Bernard Lyon 1, CNRS/IN2P3, Villeurbanne, France

36 Now at Lomonosov Moscow State University, Skobeltsyn Institute of Nuclear Physics, Moscow, Russia

${ }^{37}$ Now at CERN, Geneva, Switzerland

38 Also at Ulaanbaatar University, Ulaanbaatar, Mongolia

${ }^{39}$ Also at Department of Physics, University of Toronto, Toronto, ON M5S 1A7, Canada

${ }^{40}$ Also at LAPP, Université de Savoie, CNRS/IN2P3, Annecy-le-Vieux, France

${ }^{41}$ Now at II. Physikalisches Institut, Universität Göttingen, Göttingen, Germany

${ }^{42}$ Now at Institute for Information Transmission Problems RAS, Moscow, Russia ${ }^{1}$

43 Now at Moscow Institute of Physics and Technology, Dolgoprudny, Moscow Region, Russian Federation ${ }^{\mathrm{m}}$

${ }^{44}$ Now at Nuclear Physics Institute of the CAS, Řež, Czech Republic

45 Now at Department of Physics, Oxford University, Oxford, UK

Received: 15 June 2021 / Accepted: 23 June 2021 / Published online: 16 August 2021

(C) The Author(s) 2021

\begin{abstract}
The measurement of the jet cross sections by the $\mathrm{H} 1$ collaboration had been compared to various predictions including the next-to-next-to-leading order (NNLO) QCD calculations which are corrected in this erratum for an implementation error in one of the components of the NNLO calculations. The jet data and the other predictions remain unchanged. Eight figures, one table and conclusions are adapted accordingly, exhibiting even better agreement between the corrected NNLO predictions and the jet data.

Erratum to: Eur. Phys. J. C (2017) 77:215

https://doi.org/10.1140/epjc/s10052-017-4717-9
\end{abstract}

The original article can be found online at https://doi.org/10.1140/ epjc/s10052-017-4717-9.

ae-mail: sschmitt@mail.desy.de

${ }^{\mathrm{b}}$ Supported by the Bundesministerium für Bildung und Forschung, FRG, under contract numbers 05H09GUF, 05H09VHC, 05H09VHF, 05H16PEA

${ }^{\mathrm{c}}$ Supported by the UK Science and Technology Facilities Council, and formerly by the UK Particle Physics and Astronomy Research Council

${ }^{\mathrm{d}}$ Supported by FNRS-FWO-Vlaanderen, IISN-IIKW and IWT and by Interuniversity Attraction Poles Programme, Belgian Science Policy

e Partially Supported by Polish Ministry of Science and Higher

Education, grant DPN/N168/DESY/2009

${ }^{\mathrm{f}}$ Supported by the Ministry of Education of the Czech Republic under the project INGO-LG14033

g Supported by the Swiss National Science Foundation

${ }^{\mathrm{h}}$ Supported by CONACYT, México, grant 48778-F

i Russian Foundation for Basic Research (RFBR), grant no 1329.2008.2 and Rosatom

j Supported by the Romanian National Authority for Scientific

Research under the contract PN 09370101

${ }^{k}$ Partially Supported by Ministry of Science of Montenegro, no. $05-1 / 3-3352$

${ }^{1}$ Russian Foundation for Sciences, project no 14-50-00150

${ }^{m}$ Ministry of Education and Science of Russian Federation contract no 02.A03.21.0003

$\dagger$ Deceased.
The measurement of absolute and normalised inclusive jet and dijet cross sections by the $\mathrm{H} 1$ collaboration [1] were compared to next-to-next-to-leading order QCD predictions by the NNLOJET program [2]. An implementation error of specific integrated initial-final antenna functions that has impact on the numerical predictions for jet production cross sections in DIS was found in this numeric calculation $[3,4]$. The data, the next-to-leading (NLO) and approximate next-to-next-toleading order (aNNLO) predictions remain unchanged, as well as figures for three-jet cross sections and the results on the strong coupling constant $\alpha_{s}\left(M_{Z}\right)$, which are based on NLO predictions.

In this erratum, we provide corrected figures for the comparison of inclusive jet and dijet cross section data, both for absolute and normalised jet cross sections. The changes to the NNLO predictions are overall small, such that differences are only visible in figures of cross section ratios. The calculated values for $\chi^{2} / n_{\text {dof }}$ are corrected in Table 3 . The discussion is corrected accordingly.

\section{Cross section measurements}

The differential cross sections are presented for absolute and normalised inclusive jet, dijet and trijet production at hadron level in Ref. [1]. The agreement of the various predictions with the data is judged by calculating values of $\chi^{2} / n_{\text {dof }}$ [5]. Here $n_{\text {dof }}$ is the number of data points in the calculation. The values of $\chi^{2} / n_{\text {dof }}$ for the absolute and the normalised jet cross sections are listed in Table 3 . All calculations provide a reasonable value of $\chi^{2} / n_{\text {dof }}$, taking into account the fact that uncertainties on the theory predictions, such as scale variations or the PDF uncertainties, are not included. 
Table 3 Summary of values of $\chi^{2} / n_{\text {dof }}$ for absolute and normalised jet cross sections for the NLO, aNNLO and NNLO predictions, whenever those are available

\begin{tabular}{|c|c|c|c|c|c|c|c|}
\hline & \multirow[t]{2}{*}{$n_{\text {dof }}$} & \multicolumn{6}{|c|}{ Value of $\chi^{2} / n_{\text {dof }}$} \\
\hline & & NLO & aNNLO & NNLO & NLO & aNNLO & NNLO \\
\hline & & \multicolumn{3}{|c|}{ Absolute jet cross sections } & \multicolumn{3}{|c|}{ Normalised jet cross sections } \\
\hline Inclusive jet at low- $Q^{2}$ & 48 & 1.7 & 2.1 & 0.7 & 1.9 & 1.6 & 1.0 \\
\hline Inclusive jet at low- and high- $Q^{2}$ & 78 & 1.7 & 2.0 & 1.1 & 1.9 & 2.2 & 1.5 \\
\hline Dijet at low- $Q^{2}$ & 48 & 1.4 & 1.9 & 0.4 & 1.6 & 1.7 & 0.6 \\
\hline Trijet at low- $Q^{2}$ & 32 & 0.6 & & & 0.6 & & \\
\hline
\end{tabular}

\subsection{Inclusive jet cross section}

The measured double-differential inclusive jet cross sections as function of $P_{\mathrm{T}}^{\text {jet }}$ and $Q^{2}$ for low and high values of $Q^{2}$ are compared to different theoretical predictions in Fig. 8. Ratios of the data and of the predictions in aNNLO and full NNLO to the NLO predictions are provided in Fig. 9.

\subsubsection{Inclusive jet cross sections at low- $Q^{2}$ $\left(Q^{2}<80 \mathrm{GeV}^{2}\right)$}

The conclusions drawn on the agreement between the NNLO predictions and the data remain largely unchanged with respect to Ref. [1]. The NNLO predictions give a good description of the $P_{\mathrm{T}}^{\text {jet }}$-distributions following the excellent value of $\chi^{2} / n_{\text {dof }}$ (Table 3 ).

The NNLO corrections to the cross section predictions, which are defined as ratios of NNLO to NLO predictions and are displayed in Fig. 9, are particularly large at low values of $P_{\mathrm{T}}^{\text {jet }}$ or at low values of $Q^{2}$, equivalent to low values of the renormalisation and factorisation scales $\mu_{r}$ and $\mu_{f}$. The NNLO predictions themselves have significantly smaller scale uncertainties than the NLO predictions. At low values of $P_{\mathrm{T}}^{\text {jet }}$ and small $Q^{2}$, where the data are most precise, the uncertainties from scale variations of all predictions, however, are significantly larger than the experimental uncertainties. At higher values of $P_{\mathrm{T}}^{\text {jet }}$ the relative theoretical uncertainties are becoming smaller, but the data uncertainties, both statistical and systematic, increase and overshoot the uncertainties from scale variations.

\subsubsection{Measurement of inclusive jets at high- $Q^{2}$ $\left(Q^{2}>150 \mathrm{GeV}^{2}\right)$}

The phase space of additional inclusive jet cross sections at high values of $Q^{2}$ is extended to the region $P_{\mathrm{T}}^{\text {jet }}<7 \mathrm{GeV}$ by adding an extra bin at low $P_{\mathrm{T}}^{\text {jet }}[1]$. These additional cross section points as a function of $Q^{2}$ for inclusive jet production in the range $5<P_{\mathrm{T}}^{\text {jet }}<7 \mathrm{GeV}$ are shown in Figs. 8 and 9 .
The low- $P_{\mathrm{T}}^{\text {jet }}$ inclusive jet cross sections at high- $Q^{2}$ are underestimated by the NLO and aNNLO predictions, while the NNLO predictions give a good description of these data points. In the high- $Q^{2}$ domain the NNLO predictions have significantly smaller scale uncertainties than the NLO calculations, and the NNLO scale uncertainties typically are smaller than the experimental uncertainties. Figure 9 and the values of $\chi^{2} / n_{\text {dof }}$ in Table 3 indicate that the aNNLO predictions have difficulties describing the previously published high- $Q^{2}$ inclusive jet data [5] accurately. The NNLO predictions provide a good description of both, the low- and high- $Q^{2}$ inclusive jet data.

\subsection{Normalised inclusive jet cross section}

In order to obtain the normalised jet cross sections, cross sections for inclusive NC DIS are measured for $0.2<y<0.6$ in the $Q^{2}$ bins in the range $5.5<Q^{2}<80 \mathrm{GeV}^{2}$. The normalised inclusive jet cross sections, derived using the inclusive NC DIS and the absolute inclusive jet cross sections, are displayed together with theoretical predictions in Fig. 11. The ratio of normalised inclusive jet cross sections to NLO predictions and the predictions in aNNLO and full NNLO to the NLO predictions is shown in Fig. 12. The dominating systematic uncertainties do not cancel in the normalisation, and the systematic uncertainty is significantly reduced only in bins where the overall systematic error is small, typically at low $P_{\mathrm{T}}^{\mathrm{jet}}$. The normalised jet cross sections confronted with theoretical predictions confirm the observations obtained using the absolute cross sections.

\subsection{Dijet cross sections}

The double-differential dijet cross sections as function of $\left\langle P_{\mathrm{T}}\right\rangle_{2}$ and $Q^{2}$ are displayed in Fig. 13 and compared to theoretical predictions in NLO, aNNLO and NNLO. A comparison of the ratio of data to NLO predictions is provided in Fig. 14 together with the ratio of NNLO to NLO.

The aNNLO and NNLO predictions provide a better description of the shapes, while the NNLO predictions pro- 

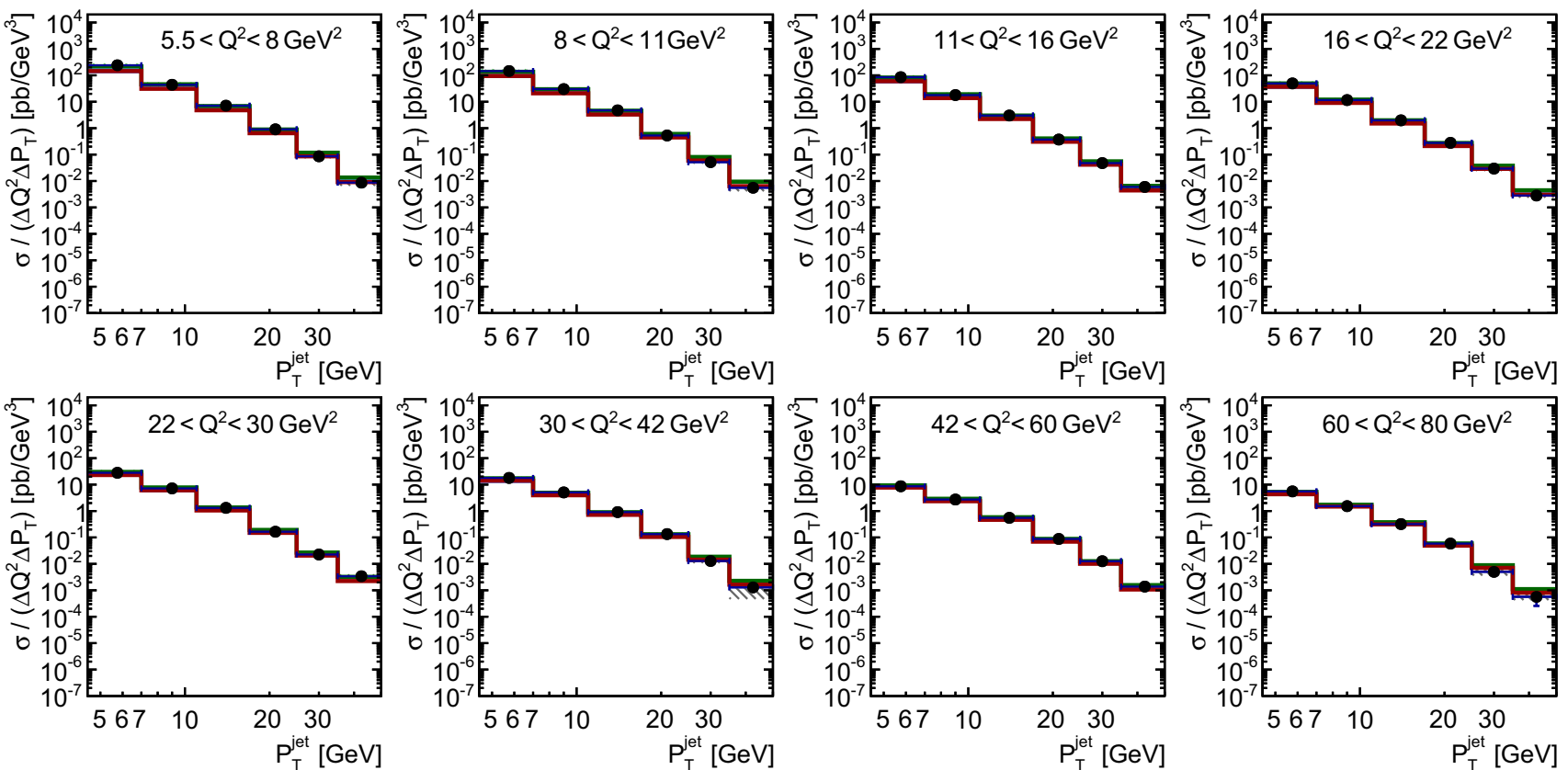

\section{$\mathrm{H} 1$ Inclusive jets}

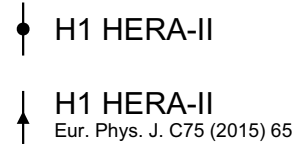

Systematic uncertainty
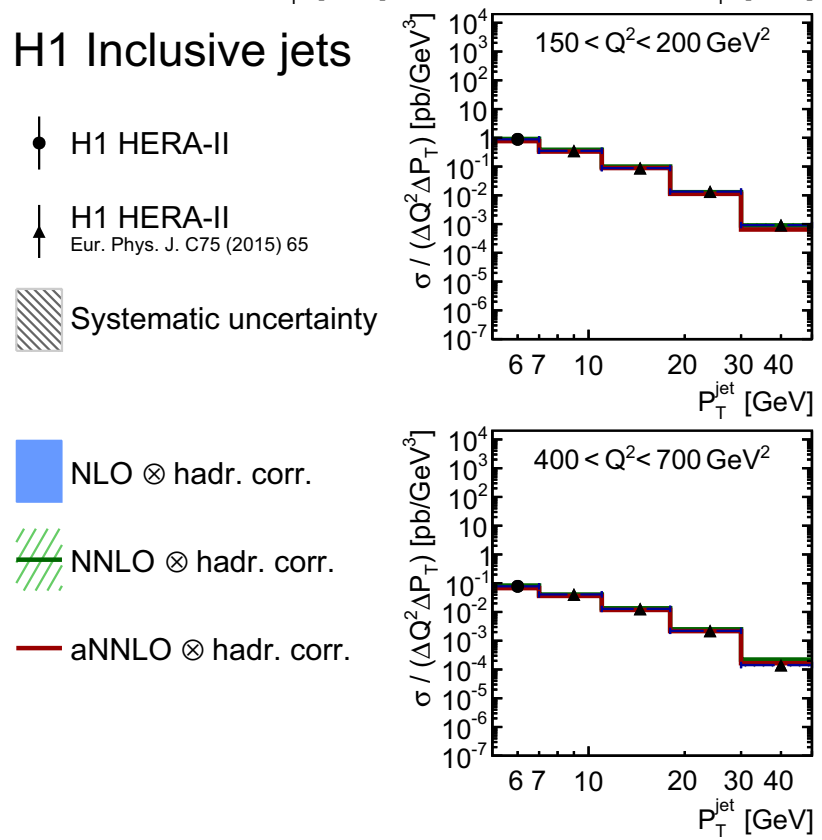

Fig. 8 Cross sections for inclusive jet production in NC DIS as a function of $P_{\mathrm{T}}^{\text {jet }}$ for different $Q^{2}$ ranges. The new data are shown as full circles whereas full triangles indicate previously published data. The error bars indicate statistical uncertainties. The hatched area indicates all other experimental uncertainties added in quadrature. The NLO and

vide an overall accurate description of the normalisation of the dijet data. The uncertainty from scale variations of the NLO predictions is larger than the experimental uncertainty for $\left\langle P_{\mathrm{T}}\right\rangle_{2}<35 \mathrm{GeV}$, while the scale uncertainty of the NNLO calculations is reduced compared to the NLO predictions and is larger than the experimental uncertainties only at lower $Q^{2}$ values and for $\left\langle P_{\mathrm{T}}\right\rangle_{2}<11 \mathrm{GeV}$.
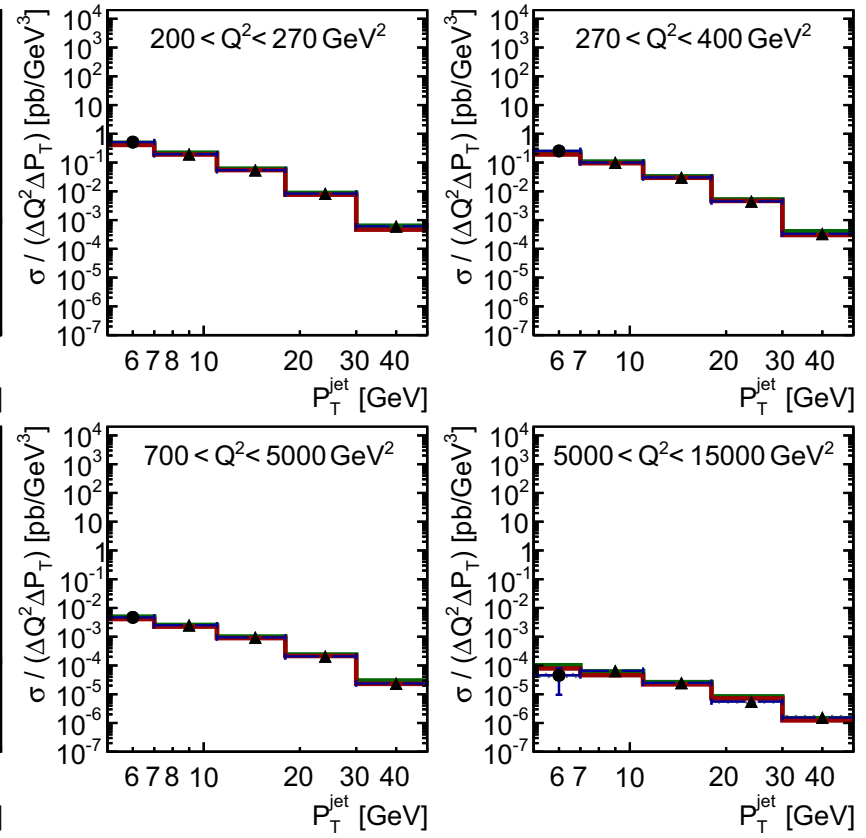

NNLO QCD predictions corrected for hadronisation effects together with their uncertainties from scale variations are shown by the shaded and hatched band, respectively. The aNNLO calculations are shown as full red line. The cross sections in each bin are divided by the bin-size in $P_{\mathrm{T}}^{\mathrm{jet}}$ and $Q^{2}$

The normalised dijet cross sections are displayed together with theoretical predictions in Fig. 15, and the ratio to NLO predictions is shown in Fig. 16. When comparing normalised dijet cross sections to theory predictions, the features observed with the absolute dijet cross sections are confirmed. 

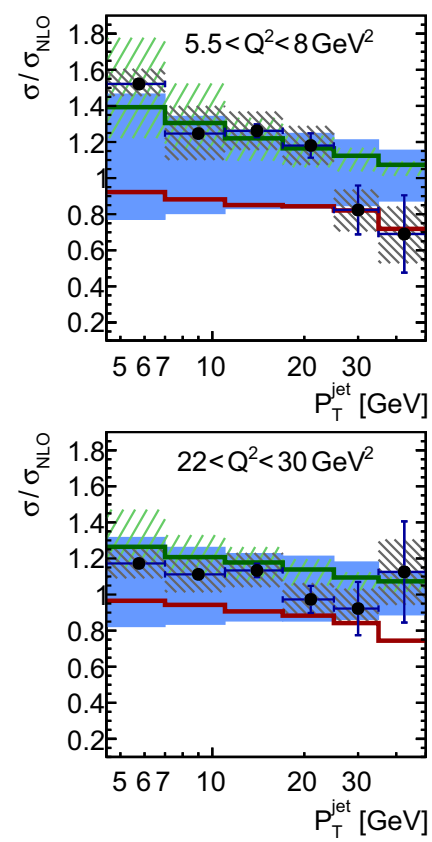

$\mathrm{H} 1$ Inclusive jets

† H1 HERA-II

| H1 HERA-II

Eur. Phys. J. C75 (2015) 65

Systematic uncertainty

$\mathrm{NLO} \otimes$ hadr. corr.

'/l/ NNLO $\otimes$ hadr. corr.

- aNNLO $\otimes$ hadr. corr.
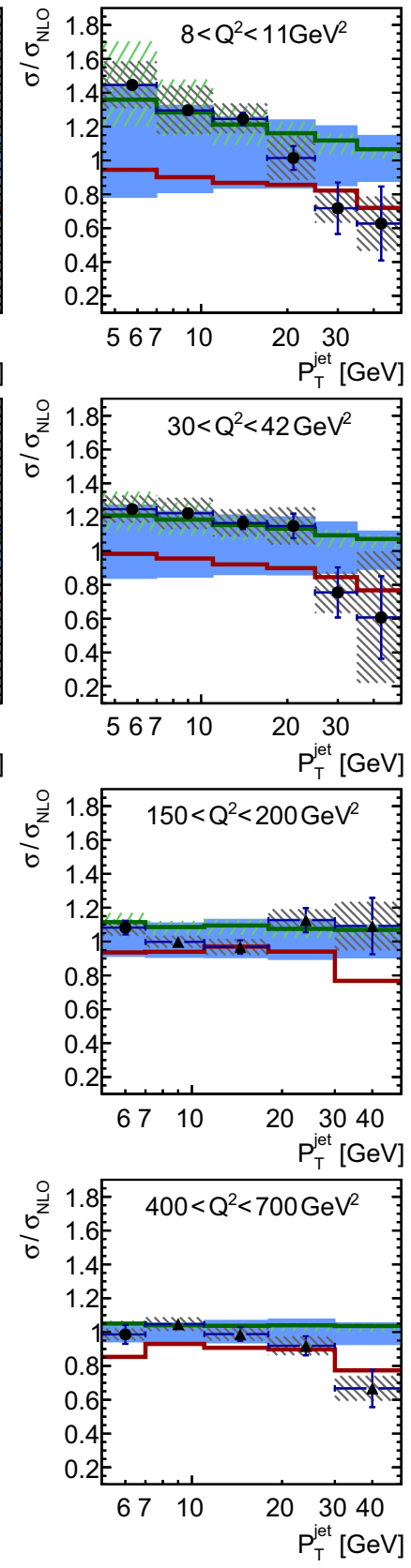
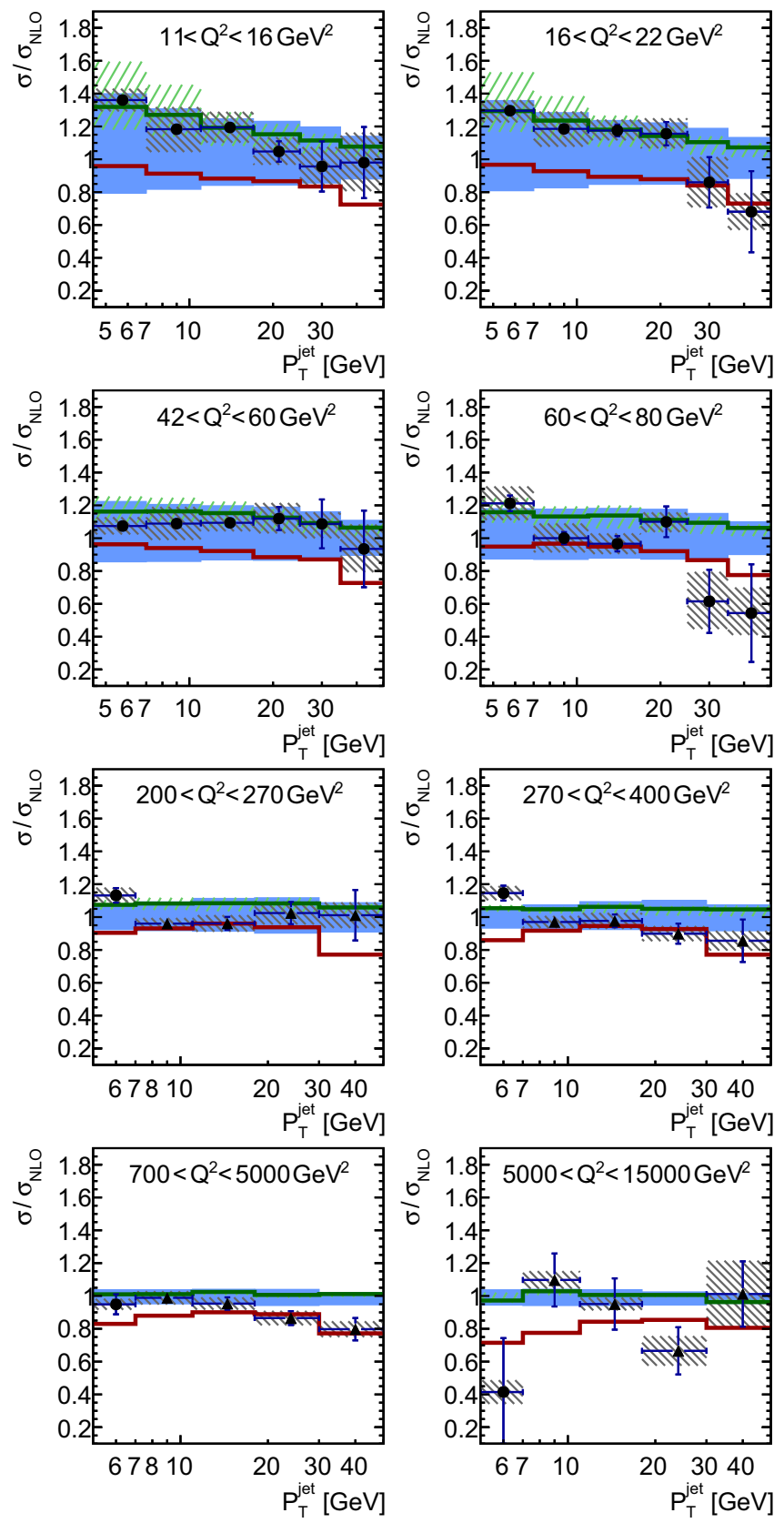

Fig. 9 Ratio of inclusive jet cross sections to the NLO predictions and ratio of aNNLO and NNLO to NLO predictions as functions of $Q^{2}$ and $P_{\mathrm{T}}^{\mathrm{jet}}$. More details are given in the caption of Fig. 8 

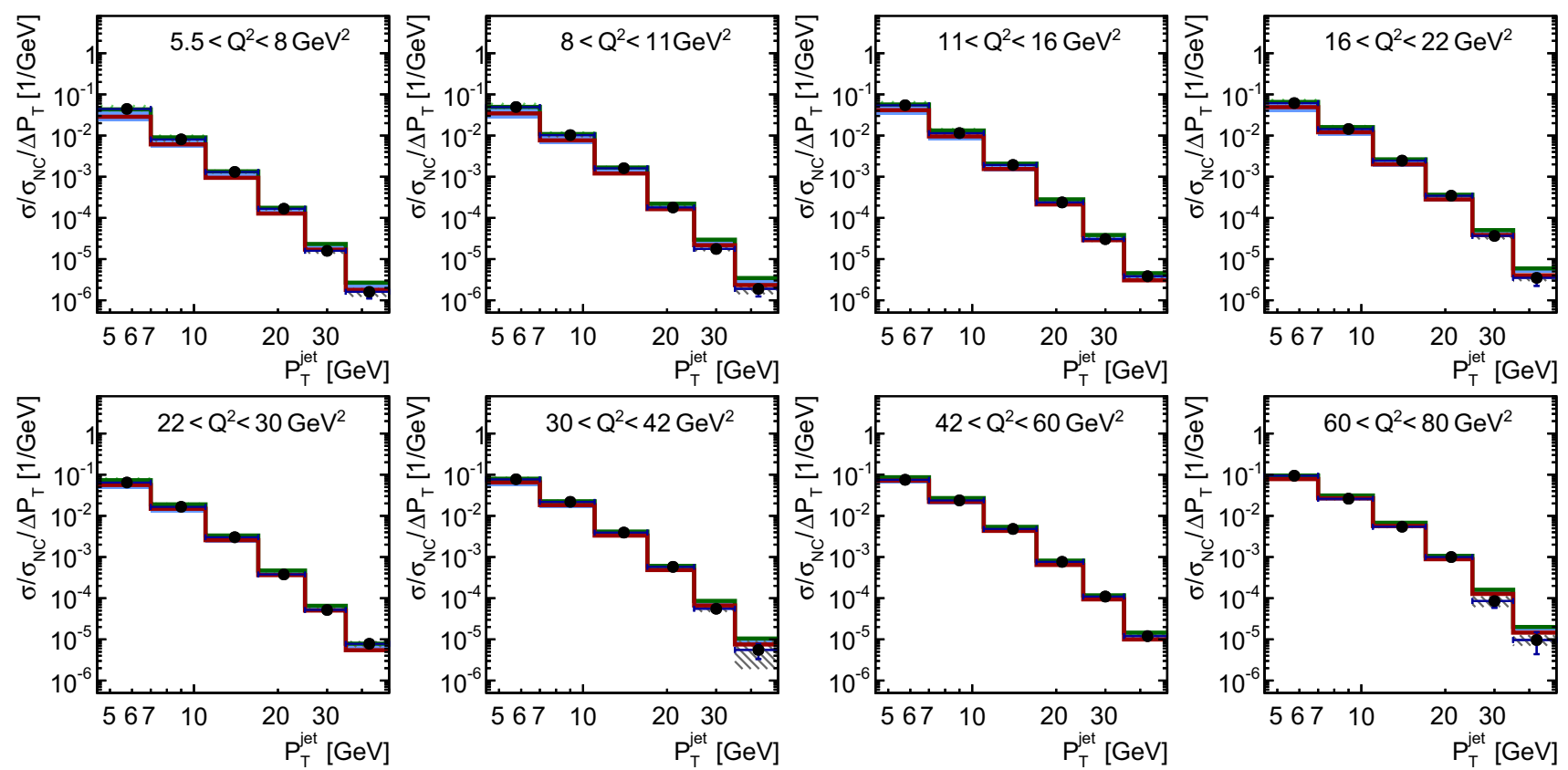

\section{H1 Normalised inclusive jets \\ † H1 HERA-II \\ $\uparrow \begin{aligned} & \text { H1 HERA-II } \\ & \text { Eur. Phys. J. C75 (2015) } 65\end{aligned}$ \\ Systematic uncertainty}

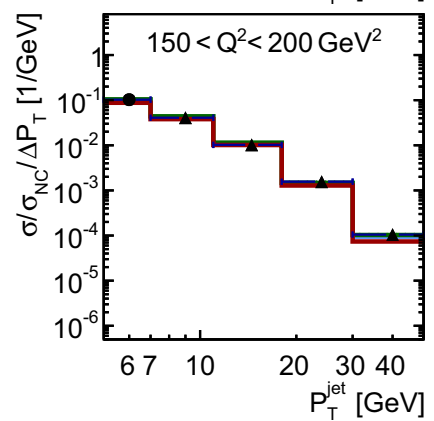

NLO $\otimes$ hadr. corr.

"WN NLO $\otimes$ hadr. corr.

— aNNLO $\otimes$ hadr. corr.

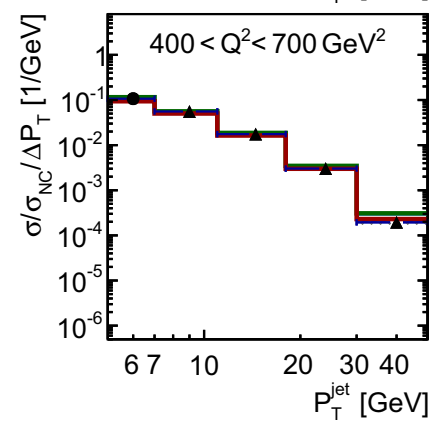

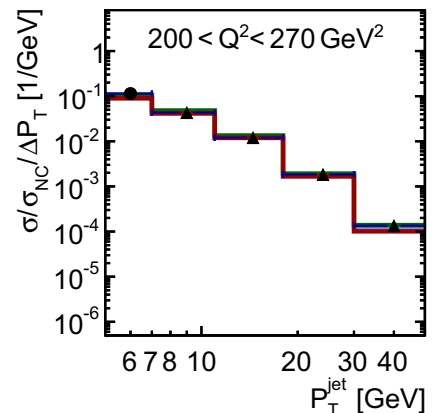
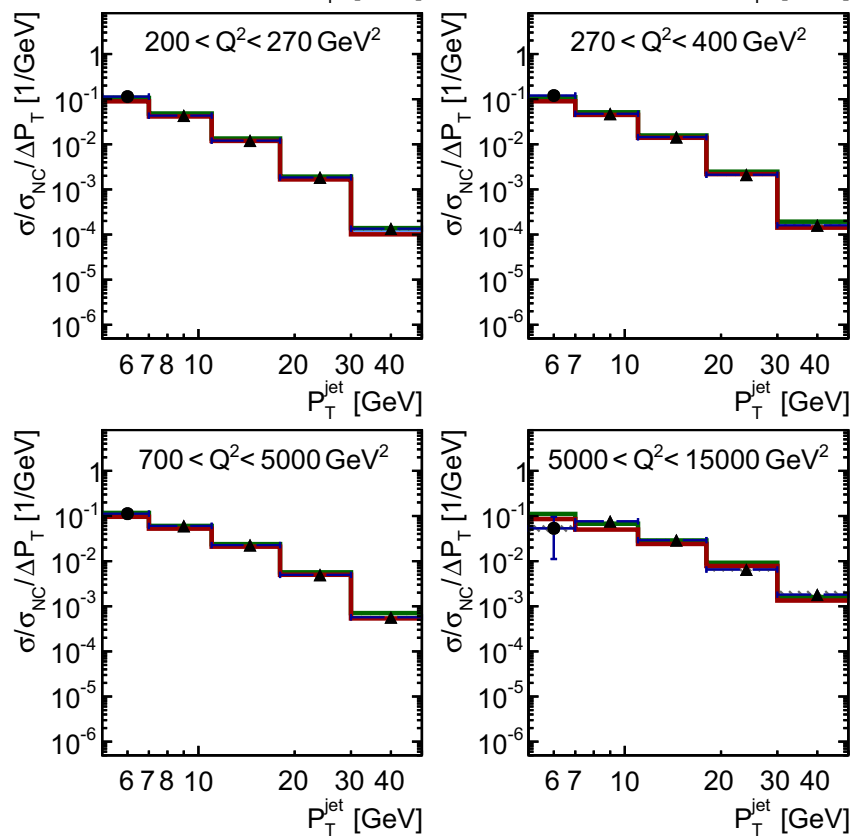

Fig. 11 Normalised inclusive jet cross sections compared to NLO, aNNLO and NNLO predictions as functions of $Q^{2}$ and $P_{\mathrm{T}}^{\text {jet }}$. The cross sections are divided in each bin by the bin size in $P_{\mathrm{T}}^{\text {jet }}$. Further details can be found in the caption of Fig. 8 

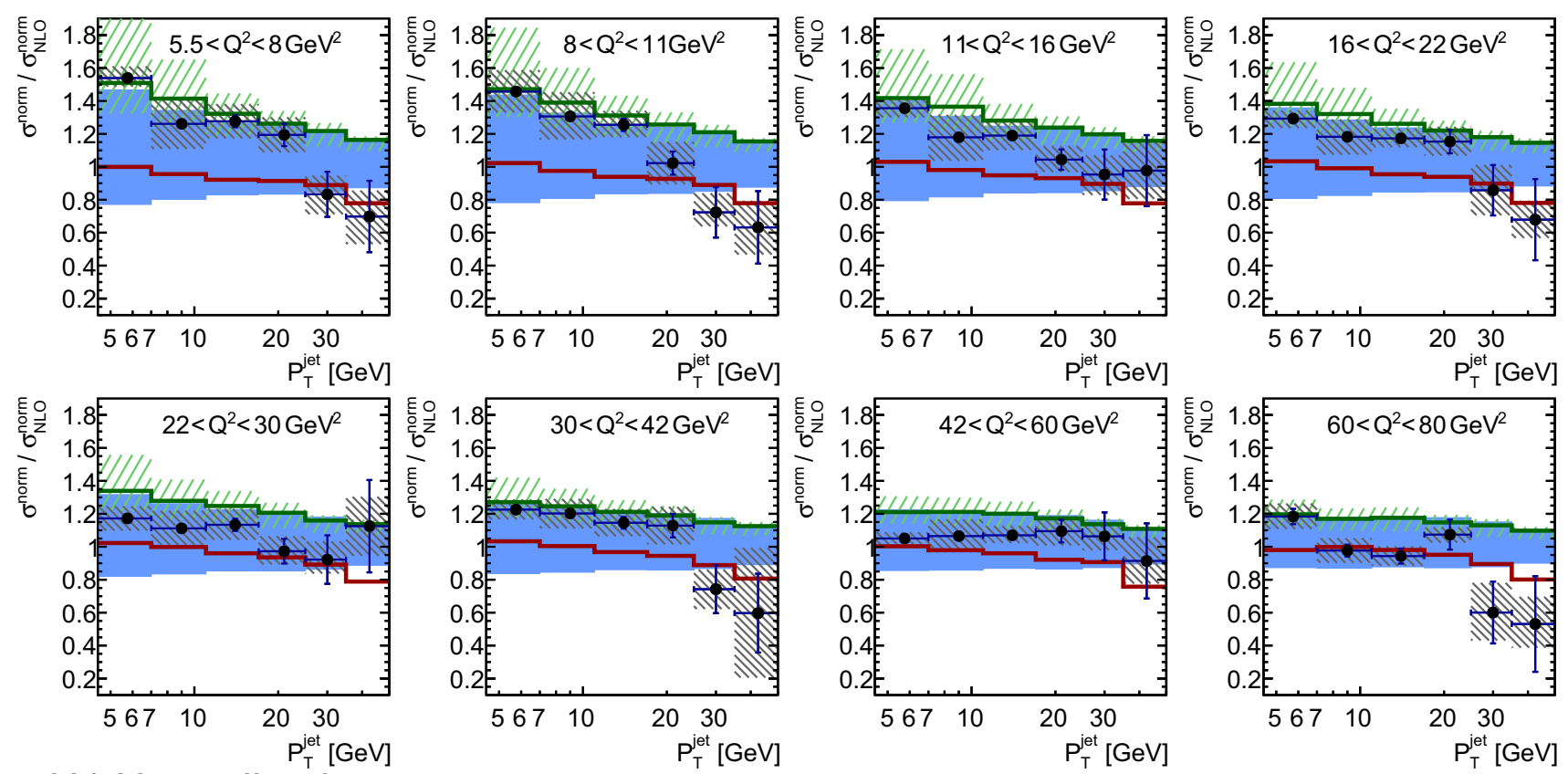

\section{H1 Normalised inclusive jets \\ H1 HERA-II \\ | H1 HERA-II \\ Eur. Phys. J. C75 (2015) 65 \\ Systematic uncertainty}
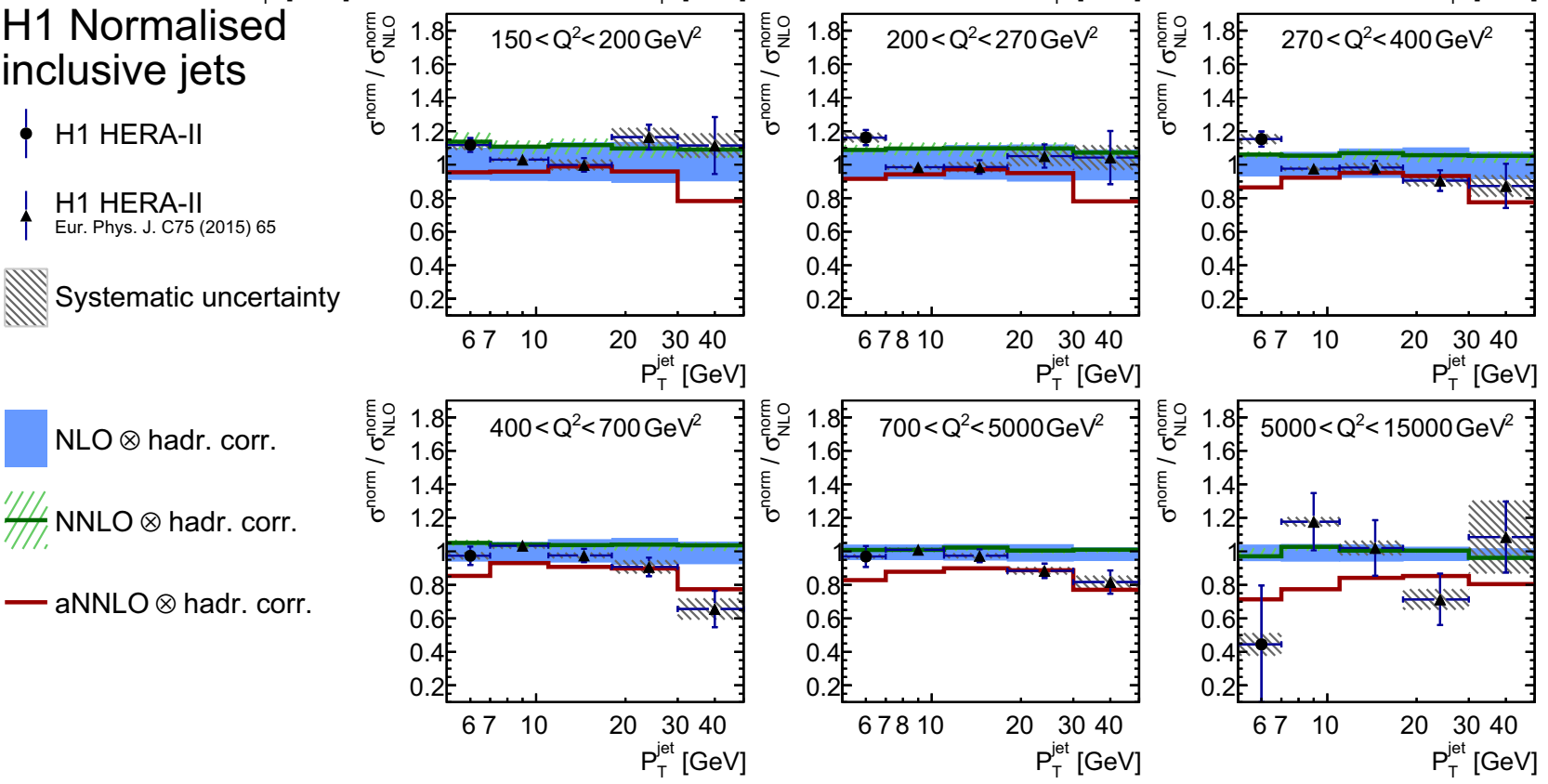

Fig. 12 Ratio of normalised inclusive jet cross sections to NLO predictions and ratio of the NNLO and aNNLO to the NLO predictions as functions of $Q^{2}$ and $P_{\mathrm{T}}^{\mathrm{jet}}$. Further details can be found in the caption of Fig. 8 
Fig. 13 Dijet cross sections compared to NLO, aNNLO and NNLO predictions as functions of $Q^{2}$ and $\left\langle P_{\mathrm{T}}\right\rangle_{2}$. The cross sections in each bin are divided by the bin-size in $\left\langle P_{\mathrm{T}}\right\rangle_{2}$ and $Q^{2}$. Further details can be found in the caption of Fig. 8
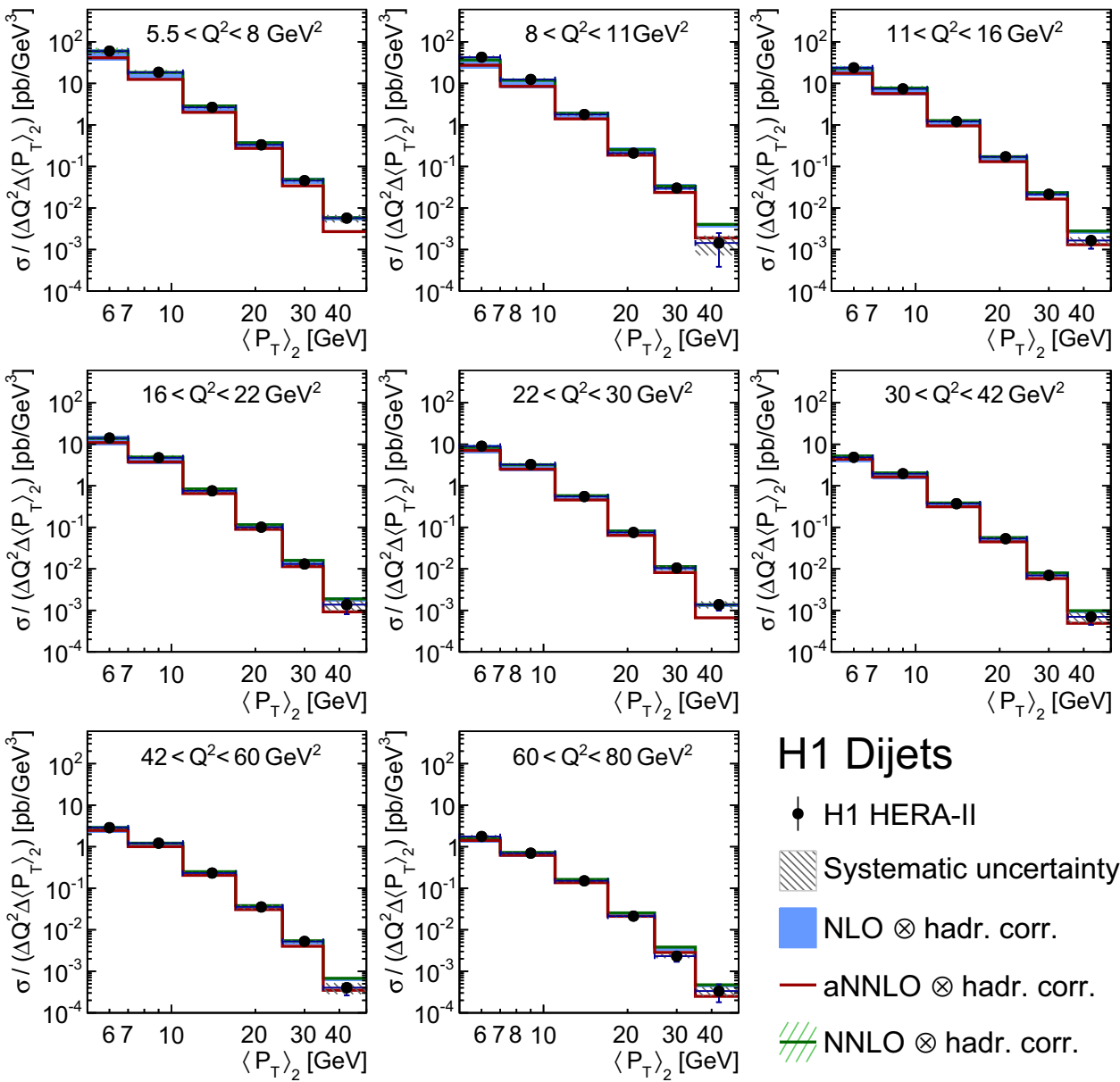

- H1 HERA-II

Systematic uncertainty

$\mathrm{NLO} \otimes$ hadr. corr.

— aNNLO $\otimes$ hadr. corr.

$\mathrm{NNLO} \otimes$ hadr. corr. 
Fig. 14 Ratio of dijet cross sections to NLO predictions and ratio of the aNNLO and NNLO to the NLO predictions as functions of $Q^{2}$ and $\left\langle P_{\mathrm{T}}\right\rangle_{2}$. Further details can be found in the caption of Fig. 8
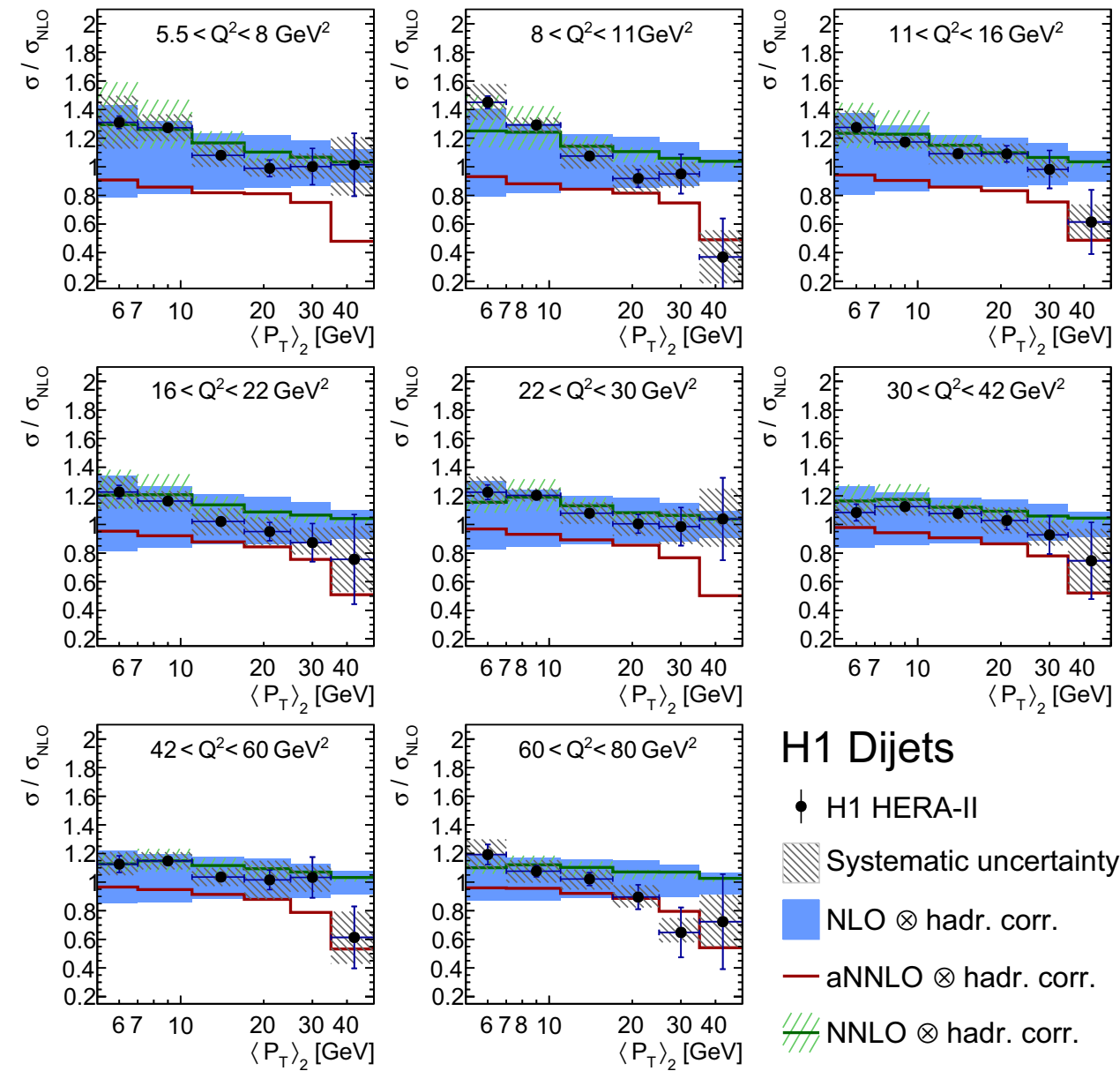

\section{H1 Dijets}

- H1 HERA-II

Systematic uncertainty

NLO $\otimes$ hadr. corr.

— aNNLO $\otimes$ hadr. corr.

NNLO $\otimes$ hadr. corr. 
Fig. 15 Normalised dijet cross sections compared to NLO, aNNLO and NNLO predictions as functions of $Q^{2}$ and $\left\langle P_{\mathrm{T}}\right\rangle_{2}$. The cross sections are divided in each bin by the bin size in $\left\langle P_{\mathrm{T}}\right\rangle_{2}$. Further details can be found in the caption of Fig. 8
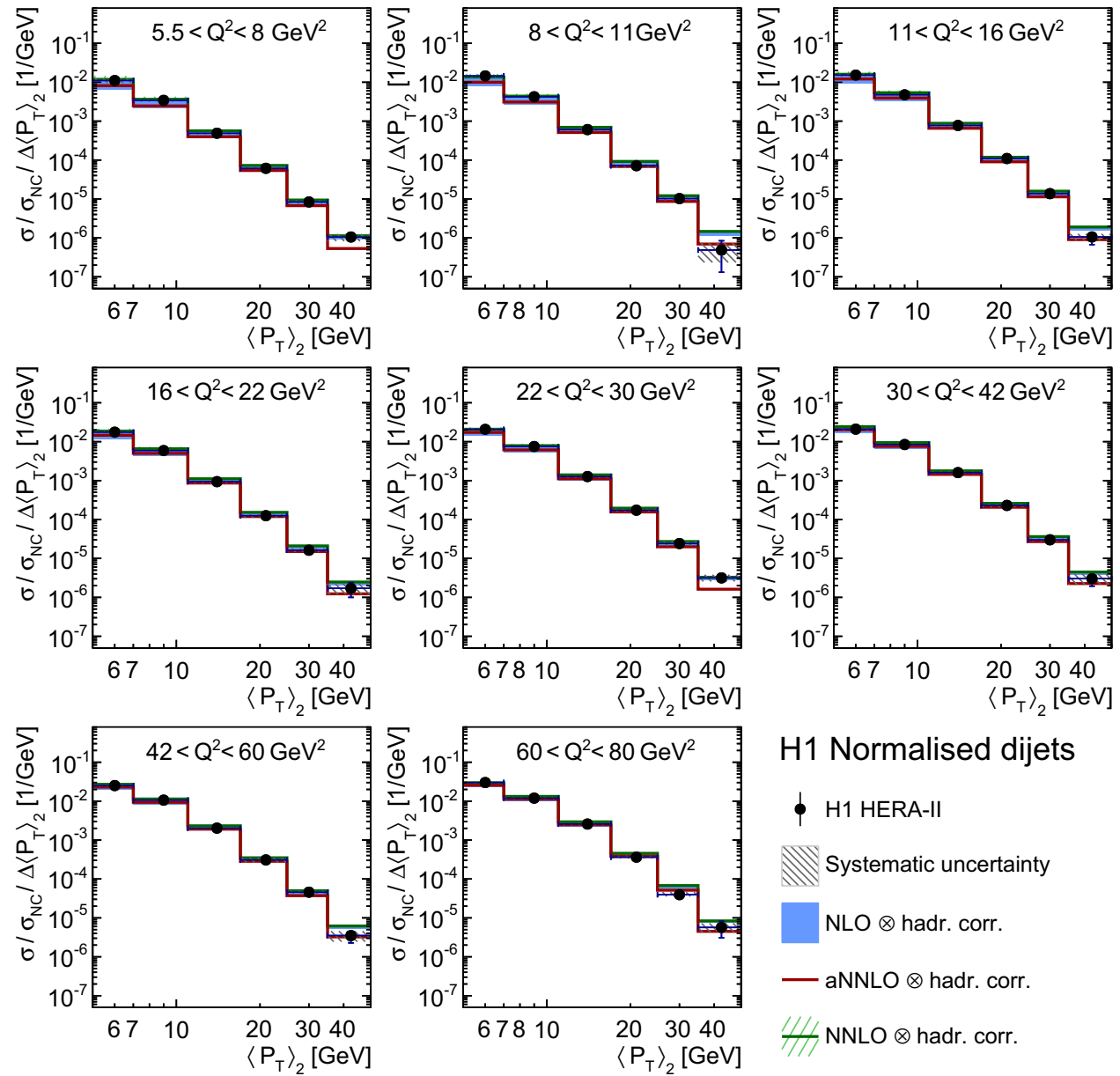

H1 Normalised dijets

H1 HERA-II

Systematic uncertainty

NLO $\otimes$ hadr. corr.

— aNNLO $\otimes$ hadr. corr.
HNLO $\otimes$ hadr. corr. 
Fig. 16 Ratio of normalised dijet cross sections to NLO predictions and ratio of the aNNLO and NNLO to the NLO predictions as functions of $Q^{2}$ and $\left\langle P_{\mathrm{T}}\right\rangle_{2}$. Further details can be found in the caption of Fig. 8
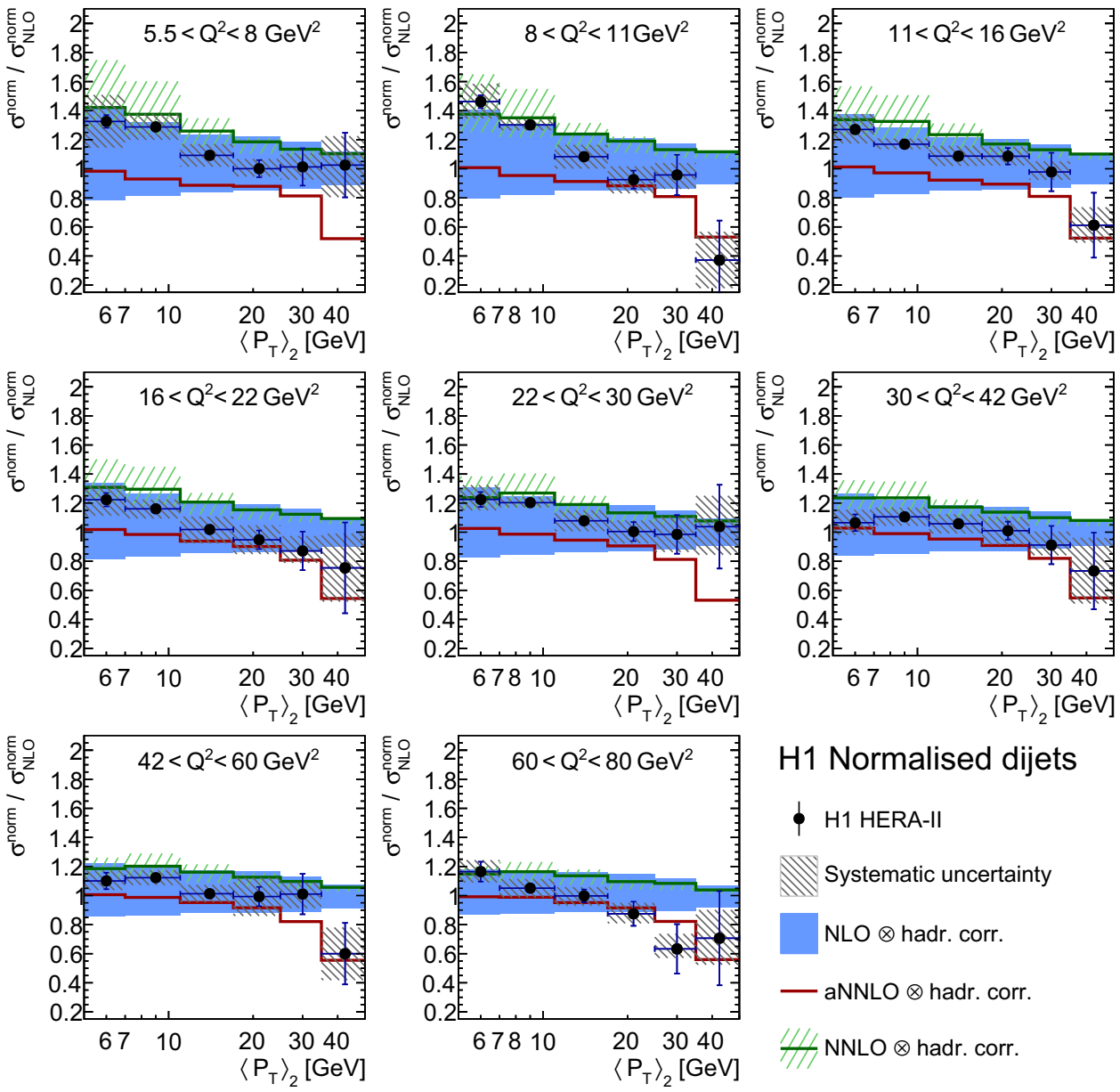

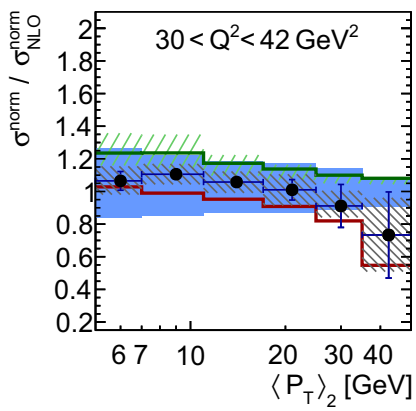

$\mathrm{H} 1$ Normalised dijets

H1 HERA-II

Systematic uncertainty

$\mathrm{NLO} \otimes$ hadr. corr.

- aNNLO $\otimes$ hadr. corr.

$\mathrm{NNLO} \otimes$ hadr. corr.

\section{Summary}

An error in the implementation of the next-to-next-to-leading order (NNLO) predictions is corrected. While the data, the NLO and the aNNLO predictions remain unchanged, some conclusions drawn from the comparison of the data to the NNLO predictions are revisited. The predictions in NNLO perturbative QCD improve the descriptions of the inclusive jet and dijet cross sections compared to NLO predictions, and give an overall good description of the new data at low and high $Q^{2}$.

Acknowledgements We thank T. Gehrmann, J. Niehues and A. Huss for providing the corrected NNLO predictions.

Open Access This article is licensed under a Creative Commons Attribution 4.0 International License, which permits use, sharing, adaptation, distribution and reproduction in any medium or format, as long as you give appropriate credit to the original author(s) and the source, provide a link to the Creative Commons licence, and indicate if changes were made. The images or other third party material in this article are included in the article's Creative Commons licence, unless indicated otherwise in a credit line to the material. If material is not included in the article's Creative Commons licence and your intended use is not permitted by statutory regulation or exceeds the permitted use, you will need to obtain permission directly from the copyright holder. To view a copy of this licence, visit http://creativecomm ons.org/licenses/by/4.0/.

Funded by $\mathrm{SCOAP}^{3}$.

\section{References}

1. V. Andreev et al., [H1 Collaboration], Eur. Phys. J. C 77, 215 (2017). arXiv: 1611.03421

2. A. Gehrmann-De Ridder et al., JHEP 1607, 133 (2016) arXiv: 1605.04295

3. J. Currie, T. Gehrmann, J. Niehues, Phys. Rev. Lett. 117, 042001 (2016). arXiv: 1606.03991

4. J. Currie, T. Gehrmann, A. Huss, J. Niehues, JHEP 1707, 018 (2017) [Erratum ibid 2012, 042 (2020)]. arXiv: 1703.05977

5. V. Andreev et al., [H1 Collaboration], Eur. Phys. J. C 75, 65 (2015). arXiv:1406.4709 